\title{
Passive generation from a novel thermoelectric energy harvesting system model integrated with phase change material
}

\author{
Yoo-Suk Byon ${ }^{1}$, Hansol Lim ${ }^{1}$, Yong-Kwon Kang ${ }^{1}$, Soo-Yeol Yoon ${ }^{1}$, and Jae-Weon Jeong ${ }^{1, *}$ \\ ${ }^{1}$ Department of Architectural Engineering, Hanyang University, Republic of Korea
}

\begin{abstract}
The purpose of this research is to evaluate the performance of a novel model that incorporates a thermoelectric generator (TEG) and phase change material (PCM). The proposed model passively generates electricity using waste heat that accumulates at exterior wall surfaces. The main generator is a TEG. To maintain the temperature difference between the two sides of the TEG, PCM is located at its cold side-thus converging the heat transferred into latent heat. The proposed passive generation system is formed into a TEG-PCM block. The block can be stacked to form a wall or inserted into any part of a building that faces the sun. The experiment setup is based on a constant temperature method. The wall temperature profile is set according to solar radiation, convection, and radiative heat transfer. To replicate daily wall temperatures during the experiment, a heat plate is used to match a wall temperature profile. Step control was used for the heating plate. The resulting data shows the average temperature difference between the hot and cold sides of the TEG to be $10-20^{\circ} \mathrm{C}$. The peak generated electricity was $0.08 \mathrm{~W}$ for a single module.
\end{abstract}

\section{Introduction}

Energy harvesting has become an important factor for newly constructed buildings. As zero-energy buildings (ZEBs) become mandatory, they will require measures that enable generating energy on their own. For a building to achieve net-zero energy, reducing loads is insufficient; buildings also need to supply their own energy. There are many methods for generating energy in buildings, but this study focuses on passive generation-in particular, harvesting waste heat accumulated on exterior walls. Many of the main technologies that are applied to ZEBs are associated with controlling heat on walls. Heat on walls has to be prevented in summer to avoid unnecessary cooling loads [1]. It is shown that, in any season, the exterior surface of a wall reaches temperatures higher than those of its indoor surface [2]. By using exterior wall heat to generate energy, buildings can utilize heat that is otherwise wasted and also reduce cooling loads.

Thermoelectric generators (TEGs) can be used to convert heat into energy. When there is a temperature difference between the hot and cold sides of a TEG, by the Seebeck effect, the TEG generates electricity. A TEG can be made into a passive electricity generator. In addition to having no moving parts, TEGs are quiet, compact, reliable, and have an operating life of over 30 years. TEGs are now being used in heat-recovering gadgets for various heat sources. Applying a heat recovery system on building walls is easy as there are many spare spaces, and substantial amounts of waste heat. However, the low conversion efficiency of TEGs is a primary concern. Because a TEG can generate electricity that will be 5\% [3] of given heat, improper heat recovery systems that include energy-consuming cooling components will indefinitely result in energy loss, rather than gain.

When using a TEG, a cooling component is required to keep one of its sides cold. This is because a TEG can only generate electricity when a temperature difference is maintained between its hot and cold sides. Former studies suggest using phase change materials (PCMs) as the cooling component. PCM can convert sensible heat into latent heat so that the required temperature difference is maintained. By using PCM as the heat sink for a TEG, no cooling energy is consumed [4].

Use of PCM in architectural engineering is focused on increasing the insulation capacity of building walls. PCM in walls can serve as a thermal barrier and heat sink. This will prevent outside heat from intruding, as well as reduce cooling loads in summer. By using PCM in the system which is installed inside exterior walls of buildings, better thermal insulation can be expected.

The proposed system incorporates a TEG and PCM to create a passive energy harvester. This energyharvesting system utilizes waste heat from the exterior walls of buildings with no moving components and no energy consumed in the process. The electricity

* Corresponding author: jjwarc@hanyang.ac.kr 
generated is expected to be used in various components within the building.

\section{TEG-PCM block}

\subsection{Thermoelectric generation module}

The TEG system basically consists of a heat source, TEG, and heat sink [3]. In this system, the main heat source is solar radiation. A black aluminum plate with an absorption rate of 0.8 acts as a heat plate that accumulates solar radiation. Other than using conservative building materials, aluminum was used to ensure better thermal conductivity to the TEG.

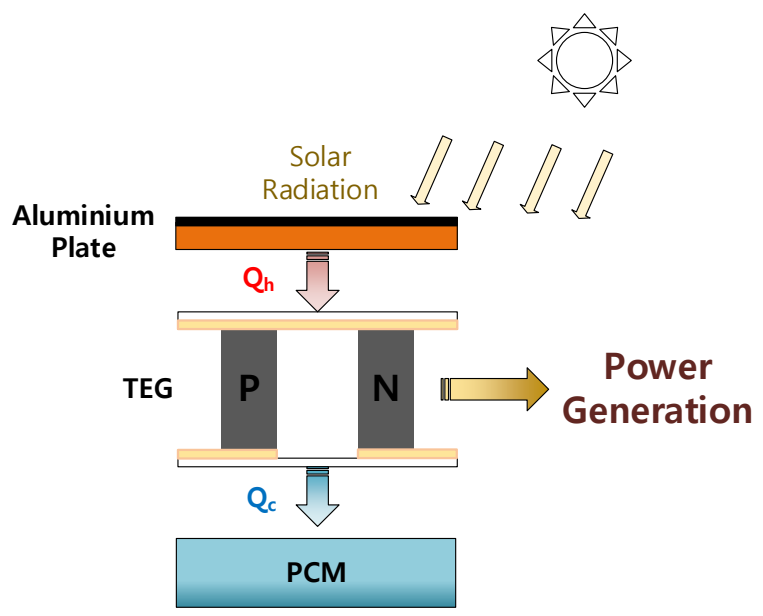

Fig. 1. Schematic of thermoelectric generation (Day).

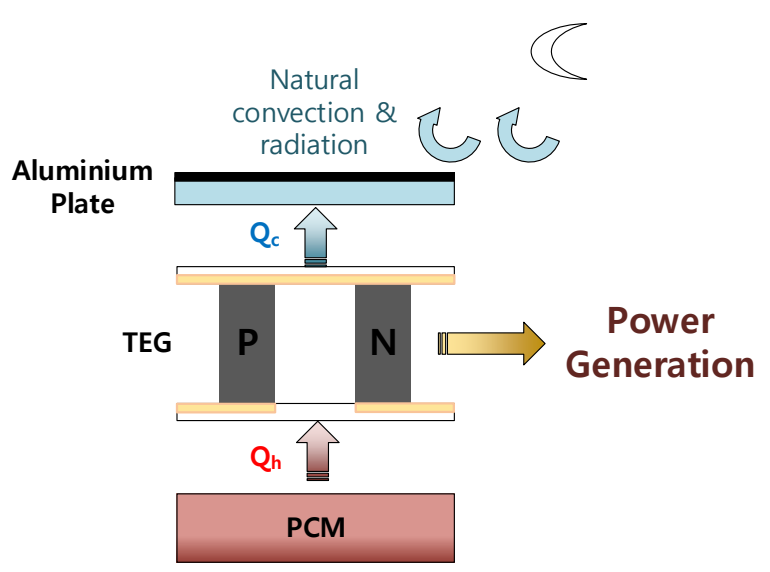

Fig. 2. Schematic of thermoelectric generation (Night).

Heat from the source is passed to the TEG, then to the heat sink (Fig. 1). PCM is used as the heat sink to provide a fixed temperature on the cold side of the TEG.

The TEG is not directionally fixed for hot and cold sides. Therefore, as long as a temperature difference exists, electricity is generated. This means that the hot and cold sides can be switched. In this system, electricity is expected to be generated throughout the day via solar radiation and stored in the PCM. At night, outdoor air temperatures drop and heat stored in the PCM travels through the TEG and is radiated outdoors. This results in continued generation over 24 hours. Although there is no cooling component to cool the PCM, the aluminum plate can work as a cooling device during the night. With the aluminum plate, the system can better radiate stored heat to the outdoors than ordinary building materials.

\subsection{Phase change material}

The PCM in the system must have a high heat capacity, be stable, and safe for use near living spaces. From various options, paraffin wax was selected for its good latent heat capacity, low cost, and long operating life [5].

The paraffin wax in the proposed system is Parafol 17-97 (n-heptadecane) with a melting point at $22^{\circ} \mathrm{C}$. Table 1 shows the properties of Parafol 17-97. Due to its pin-like molecular structure, Parafol 17-97 is highly permeable and must be contained in an aluminum case or latex bag. Otherwise, if put in containers made of vinyl or with loose edges, it will leak. Capsulizing is one way to contain Parafol 17-97 safely. However, since PCM in the proposed system must be thermally accessible, capsulizing is not an option. In this study, a custom-made aluminum case with densely closed edges was implemented.

Table 1. Properties of Parafol 17-97.

\begin{tabular}{|c|c|}
\hline Property & Value \\
\hline Name & Parafol 17-97 \\
\hline Name of the chemical compound & n-heptadecane \\
\hline Melting point $\left({ }^{\circ} \mathrm{C}\right)$ & 22 \\
\hline Density $(\mathrm{kg} / \mathrm{L})$ & 0.77 \\
\hline $\begin{array}{c}\text { Latent heat capacity } \\
(\mathrm{kJ} / \mathrm{kg})\end{array}$ & 0.2 \\
\hline Liquid conductivity $(\mathrm{W} / \mathrm{m} \cdot \mathrm{K})$ & 0.15 \\
\hline Solid conductivity $(\mathrm{W} / \mathrm{m} \cdot \mathrm{K})$ & $2.24-2.34$ \\
\hline $\begin{array}{c}\text { Liquid Specific heat } \\
(\mathrm{kJ} / \mathrm{kg} \cdot \mathrm{K})\end{array}$ & $1.77-1.86$ \\
\hline $\begin{array}{c}\text { Solid Specific heat } \\
(\mathrm{kJ} / \mathrm{kg} \cdot \mathrm{K})\end{array}$ & \\
\hline
\end{tabular}

The main drawback of Parafol 17-97 is its low conductivity (see Table 1). The proposed system must actively exchange heat between the heat source and heat sink, and vice versa. Therefore, for the TEG-PCM module, heat pipe was installed inside the aluminum PCM casing to encourage heat transfer.

\subsection{TEG-PCM block design}

\subsubsection{TEG-PCM module}

Figure 3 depicts the TEG-PCM module. The aluminum case containing PCM is attached to the TEG with thermal grease - this is crucial to distribute heat evenly. Heat pipes are installed inside the case to facilitate heat distribution from the TEG to the PCM, and the aluminum case itself acts as a secondary thermal bridge that transfers heat to the far side of the TEG. The PCM 
surface that contacts the casing and heat pipes has to be large so as to prevent unbalanced melting. When PCM melts, it becomes liquefied and is no longer able to store heat as latent heat. Rather, heat transferred from the TEG is stored in liquid PCM in the form of sensible heat. This increases the temperature of the TEG's cold side. Maximizing the PCM surface slows down the overall liquefying effect. By simulation, it was confirmed that the thicker the liquid PCM, the hotter the cold side becomes. Therefore, two heat pipes were installed.

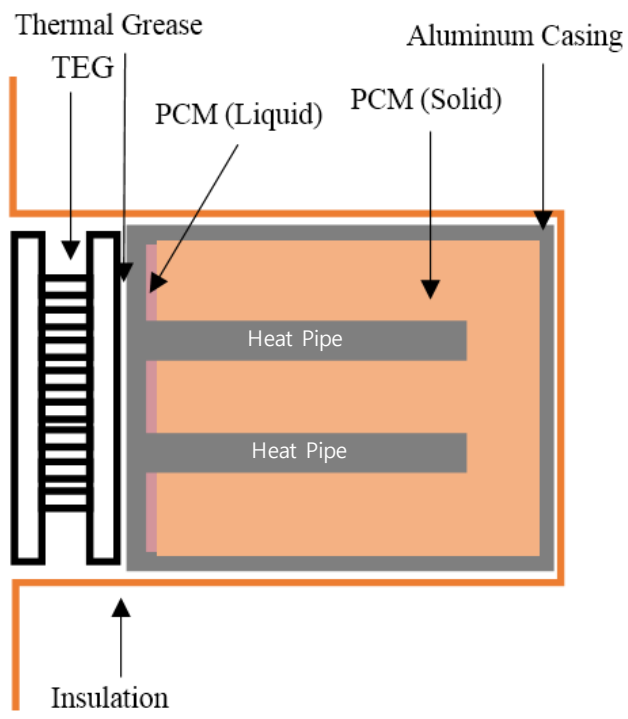

Fig. 3. Section of the TEG-PCM module.

\subsubsection{TEG-PCM block and applications}

The proposed TEG-PCM module is designed to be integrated into building blocks to form a TEG-PCM block (Figure 4). The topic of this study is harvesting exterior wall waste heat. Building block was selected to encompass the TEG-PCM module as it is small, modular, and easy to extend. This basic wall component can be installed in any wall - or used exclusively to form one.

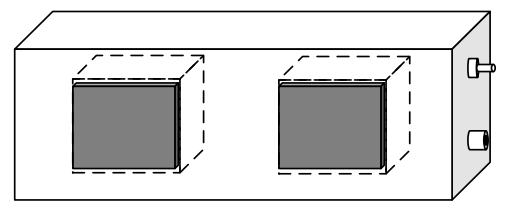

Fig. 4. The TEG-PCM block

When TEG-PCM blocks are forming a wall, they have to be electrically connected so as to store meaningful power. The block was designed to have plugs on each side. This is because prevailing blocks are stacked and glued with cement. By connecting blocks by their plugs at the sides, ordinary block-stacking will not be hindered.

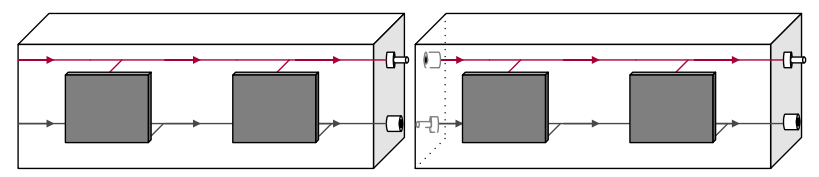

Fig. 5. TEG-PCM block connections

Blocks in the same row form a serial series, while other rows are connected as parallel series. The collected power is stored in a battery to then be used for regional devices, such as sensors and controllers, or collected for devices that require higher power to operate.

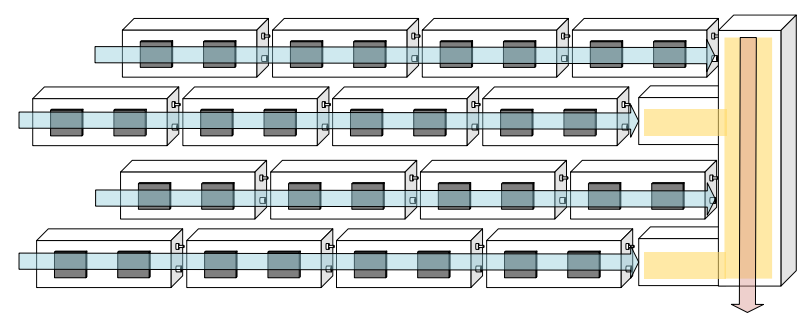

Fig. 6. TEG-PCM block wall

This chapter has described the fundamental design of the TEG-PCM block and its applications. The main theme of the design is being small, modular, and thus applicable. Since the main heat source is waste heat from exterior walls, the following experiment was based on the geometry of the TEG-PCM block standing vertically, as shown in Figure 4.

\section{Experimental setup}

\subsection{Experimental conditions}

Considering solar radiation as the heat input is slightly inaccurate, although the main heat source for the TEGPCM system is solar radiation. The collective exterior heat that incorporates additional environmental properties - such as outdoor temperature, wall-to-air heat transfer coefficient, and wall absorptivity-has to be considered. As such, a wall temperature profile was calculated by the sol-air temperature method.

\subsubsection{Sol-air temperature}

Sol-air temperature can predict exterior wall temperatures according to given environmental conditions [6]. Equation (1) is used to calculate sol-air temperature. The wall temperature is given by outdoor air temperature $\left(T_{\mathrm{OA}}\right)$, hourly solar radiation $\left(I_{\mathrm{t}}\right)$, absorptivity $(a)$, and heat transfer coefficient for radiation and convection $\left(h^{-1}\right)$. Outdoor air temperature (Seoul, Korea) and hourly solar radiation were based on annual average weather data collected by the Korean Solar Energy Society [7]. The acquired horizontal solar radiation was converted into vertical solar radiation using Engineering Equation Solver (EES). The absorptivity of the outermost component - in this case, the black aluminum plate-is 0.8 . The collective heat transfer coefficient for radiation and convection is 15 $\mathrm{W} / \mathrm{m}^{2} \cdot \mathrm{K}$ - acquired from a previous study about heat transfer coefficient at building surfaces [8].

$$
T_{\text {sol-air }}=T_{\mathrm{OA}}+I_{\mathrm{t}} \times a \times h^{-1}
$$




\subsubsection{Wall temperature profile}

Using sol-air temperature, an annual wall temperature profile for a design building was calculated (Figure 7). The wall temperature profile in Figure 8 indicates how much heat is accumulated on exterior walls, and thus an experimental model can be derived. The experiment was conducted based on the temperature difference described in the wall temperature profile. The amount of heat after sunrise is shown by the rise in wall temperature.

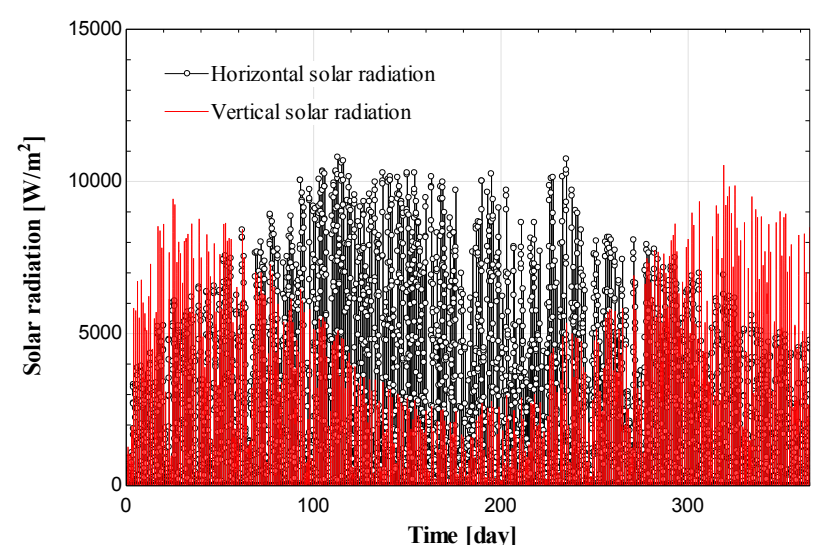

Fig. 7. Horizontal and vertical solar radiation

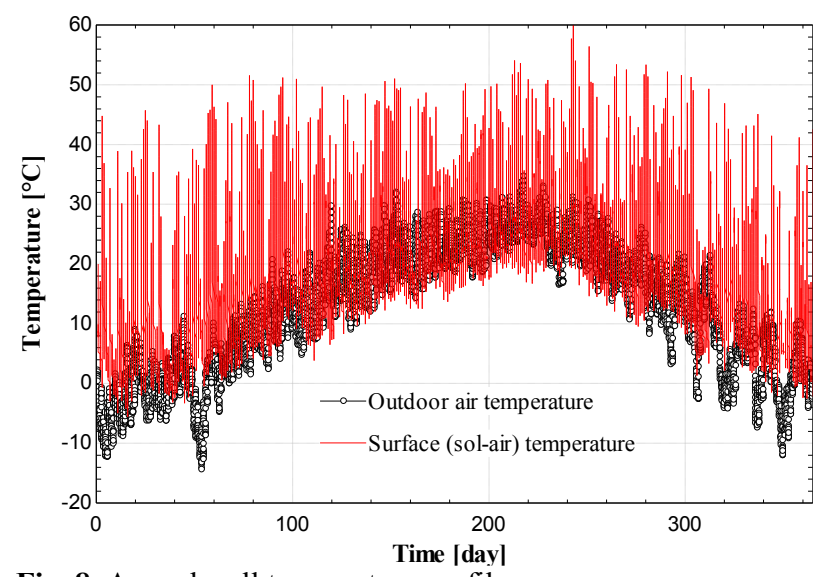

Fig. 8. Annual wall temperature profile

As explained in Section 2.3.2, the TEG-PCM system is designed to stand vertically (i.e., the TEG stands vertically). Figure 7 shows the difference between horizontal and vertical solar radiation. Vertical solar radiation is less in summer and more in winter. This results in greater wall temperature fluctuations in winter. Throughout the year, wall temperature rises as the wall receives solar radiation. The temperature then drops to outdoor air temperature at night, giving the PCM an opportunity to relinquish stored heat to the outdoors.

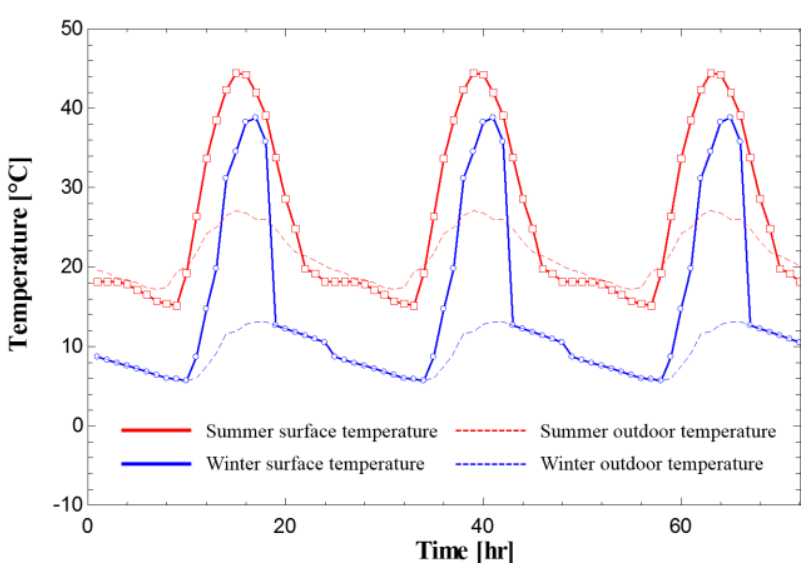

Fig. 9. Representative profile for winter and summer

Figure 9 shows the representative wall temperature profile across 72 consecutive hours in winter and summer. The dotted lines indicate outdoor temperatures. The solid blue and red lines indicate the sol-air temperatures during winter and summer, respectively. The graph shows that convective and radiative heat transfer on building walls make wall temperatures rise $20-30^{\circ} \mathrm{C}$. Based on these findings, an experiment was carried out. PCM was cooled to $10-15^{\circ} \mathrm{C}$ and then heated with a $20-30^{\circ} \mathrm{C}$ temperature difference.

\subsection{Experimental setup}

The experimental setup consists of the TEG-PCM module, components for heating and cooling, and sensors (Fig. 10.). Heating and cooling components consist of a constant temperature plate, cooling water, and a chiller to manage the water temperature. The constant temperature plate is made of 4 thermoelectric modules (TEMs) and mimics the heated exterior wall. Since the constant temperature plate is made of TEMs, it also serves as the cooling plate, mimicking lower temperatures at night. The constant temperature plate controls the surface temperature based on a set temperature and has a range of $-20^{\circ} \mathrm{C}$ to $60^{\circ} \mathrm{C}$.

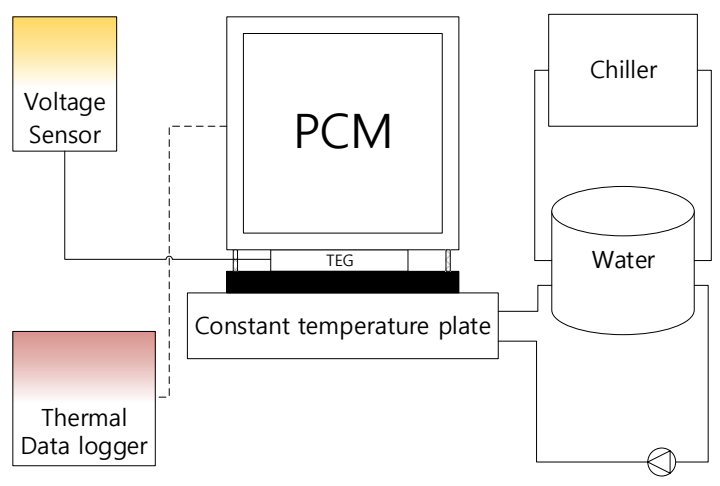

Fig. 10. The experimental setup schematic 


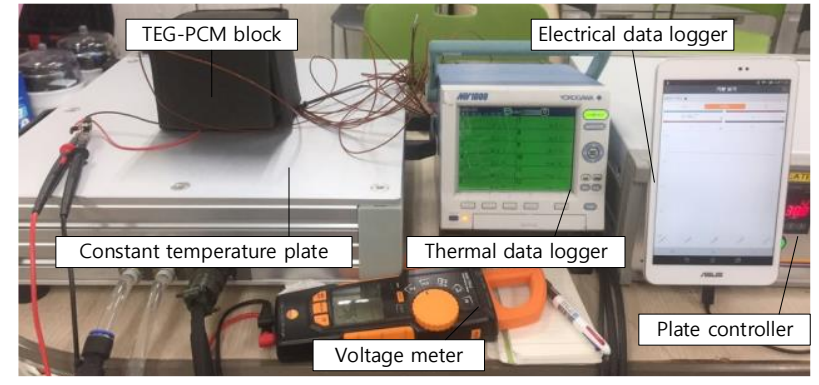

Fig. 11. Picture of the experimental setup

There are two sensors. The first is the TESTO 770-3 voltage meter shown at the bottom of Figure 11. It measures open-circuit voltage in order to determine the generated voltage contour. The second sensor is the YOKOGAWA MV2000 thermal data logger. There are a total of 6 thermocouples attached at the TEG-PCM module (Figure 12): 'Black 1' is installed for tracing the exterior temperature change; 'TEG hot' and 'TEG cold' are for the two sides of the TEG and are directly related to generated voltage; those at 'PCM 1', 'PCM 2', and 'PCM 3' are installed to predict the PCM temperature. It is best when thermocouples touch the PCM, but due to the tendency of Parafol 17-97 to leak, the thermocouples had to be attached to the nearest aluminum case.

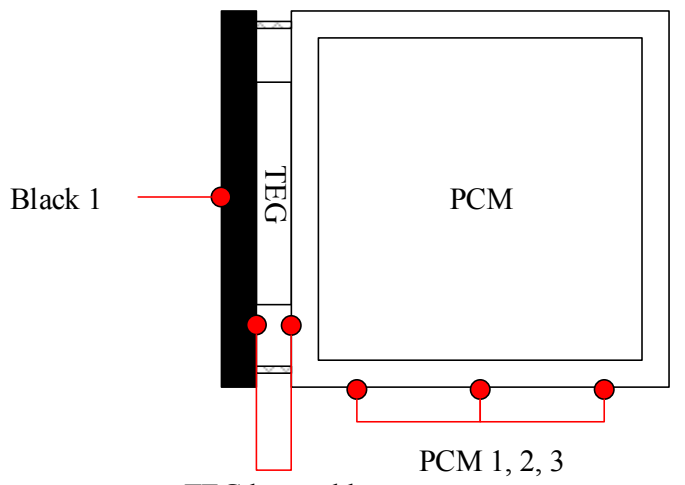

TEG hot, cold

Fig. 12. Attached thermocouples

\section{Results}

The following results show the temperature change and generated voltage. In accordance with Section 3.2.1, experiments for winter and summer conditions were conducted (Table 2). The experiment was to observe both the temperature change of the system when it was heated, as well as the resulting power generation in regard to the voltage change.

Table 2. Experimental conditions

\begin{tabular}{|c|c|c|}
\hline Season & $\begin{array}{c}\text { Starting } \\
\text { temperature }\left({ }^{\circ} \mathbf{C}\right)\end{array}$ & $\begin{array}{c}\text { Heating } \\
\text { temperature }\left({ }^{\circ} \mathbf{C}\right)\end{array}$ \\
\hline Winter & 10 & 30 \\
\hline Summer & 15 & 45 \\
\hline
\end{tabular}

\subsection{Thermal results}

The results show that as the TEG-PCM block is heated, its cold side is successfully being cooled by the PCM. Whereas the temperature of 'TEG hot' follows the surface temperature, that of 'TEG cold' is maintained near that of 'PCM'.

Both results show slower temperature change near the melting point of Parafol $17-97\left(22^{\circ} \mathrm{C}\right)$. This is when the PCM receives heat from the surface and converts it into latent heat. However, the results also show that the more drastic temperature differences in the summer condition caused the PCM to degrade more rapidly (Fig. 13. and14.). Because the volume of PCM is the same for each condition, the amount of latent heat it can store is limited and the same. Although the PCM melted sooner in the summer condition experiment, it showed a larger temperature difference between the hot and cold sides of the TEG-which is proportional to voltage generation.

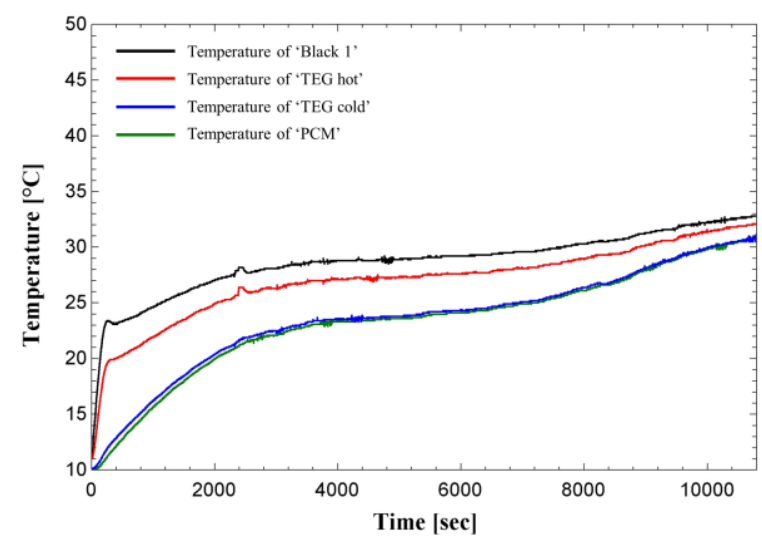

Fig. 13. TEG-PCM temperature change in winter cond.

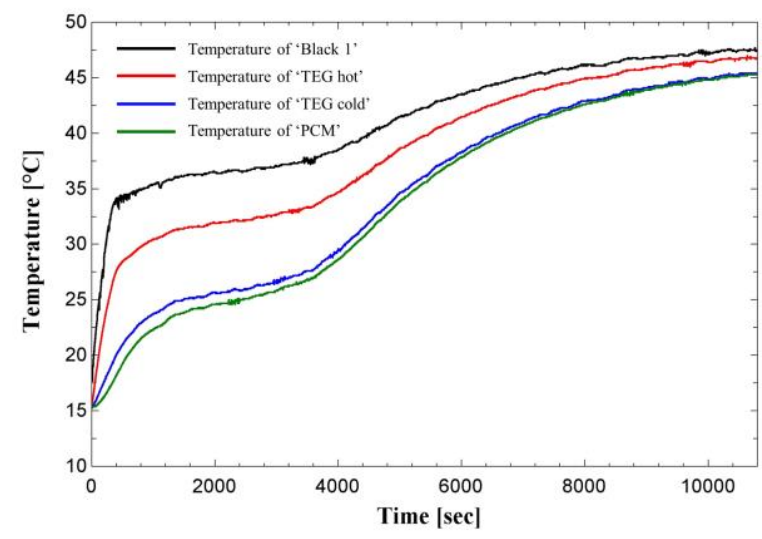

Fig. 14. TEG-PCM temperature change in summer cond. 


\subsection{Generated electricity}

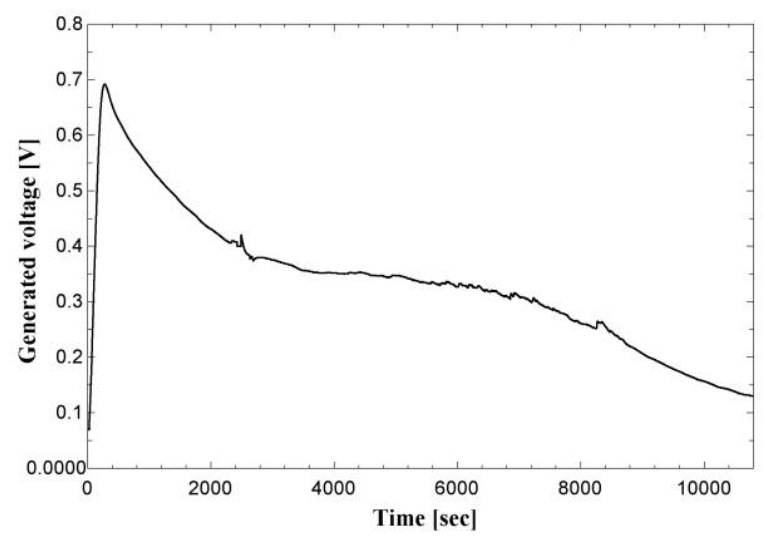

Fig. 15. Generated Voltage change in winter condition.

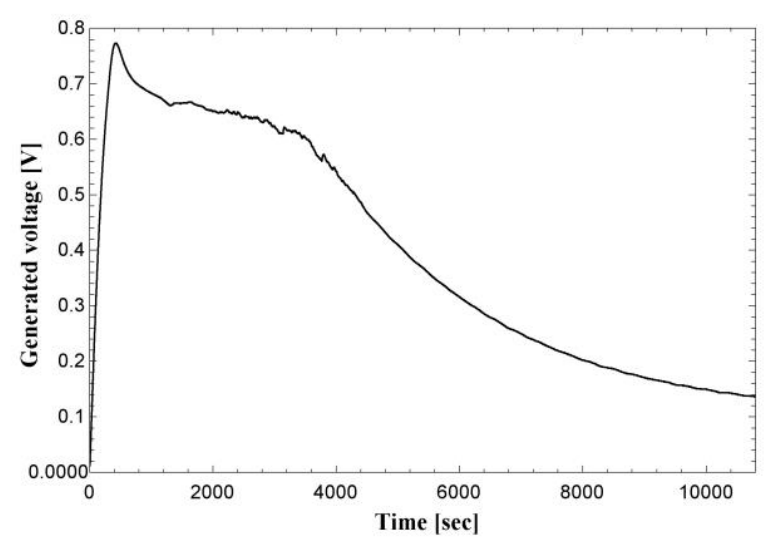

Fig. 16. Generated Voltage change in summer condition.

The generated voltage showed difference in between experiments in two conditions. The voltage dropped to a meaningless value of 0.1 at the same time, but peak voltage generated was higher in the summer condition (Fig. 15. and 16.). This is because the peak temperature difference in the TEG was greater in the summer condition. However, with a lower peak voltage, the winter condition experiment generated more stable electricity as the PCM was not fully melted for a longer period.

The overall contour of generated voltage followed the thermal results. Where the temperature difference in the TEG was greater, so was the generated voltage. Conversely, when the PCM was fully melted and the hot and cold sides of the TEG reached the same temperature, electricity generation ceased.

\section{Conclusions}

By conducting experiments with the TEG-PCM block, it could be seen that electricity was able to be generated without any extra energy given to the system. With PCM passively cooling one side of the TEG, a temperature difference was maintained that resulted in electricity generation. Based on this feasibility check, future works will be focused on scaling the electricity generation to usable amount in consideration with generated voltage and power.

Wall temperature changes of $20-30^{\circ} \mathrm{C}$ helped the TEG-PCM block generate a peak voltage of $0.8 \mathrm{~V}$. With an average current of $0.1 \mathrm{~A}$, peak generated power was 0.08 . The experiment showed that with greater temperature differences, electricity was generated more rapidly; but as the PCM melted quickly, the generation ended sooner. It was found that the balance between rapid and stable generation must be investigated.

Furthermore, the PCM depleted sooner than expected. This is easier to control with a constant temperature method, but with the PCM receiving heat from the surface, more heat flux than in a real situation was given. The wall temperature profile needs to be modified regarding heat transfer to the PCM.

This work was supported by the Korean Agency for Infrastructure Technology Advancement (KAIA) grants (19CTAP-C141826-02), and by the Korean Institute of Energy Technology Evaluation and Planning (KETEP) (No. 20184010201710).

\section{References}

1. S. M. Al-Masrani, K. M. Al-Obaidi, N. A. Zalin, M. I. Aida Isma, Solar Energy, 170 : 849-872 (2018)

2. Y. Tham, T. Muneer, Building and environment, 46 : 1243-1250 (2011)

3. S. B. Riffat, X. Ma, Appl therm eng, 23 : 913-935 (2003)

4. M. Jaworski, M. Bednarczyk, M. Czachor, Appl Therm Eng, 96 : 527-33 (2016)

5. M. M. Farid, A. M. Khudhair, S. A. K. Razack, S. Al-Hallaj, Energy conversion \& management, 45 : 1597-1615 (2018)

6. K. A. Al-Saud, Arch. \& planning, 21 : 1-7 (2009)

7. The Korean solar energy society, Standard weather data for Korean cities (2015)

8. A. Hagishima, J. Tanimoto, Building and environment, 38 : 873-881 (2003) 